\title{
Aplicabilidade do método de Ball para o cálculo da letalidade de processos de esterilização em autoclaves a vapor desaeradas por água
}

\author{
Applicability of the Ball method in the calculation of lethality \\ in sterilization processes using water vented steam retorts
}

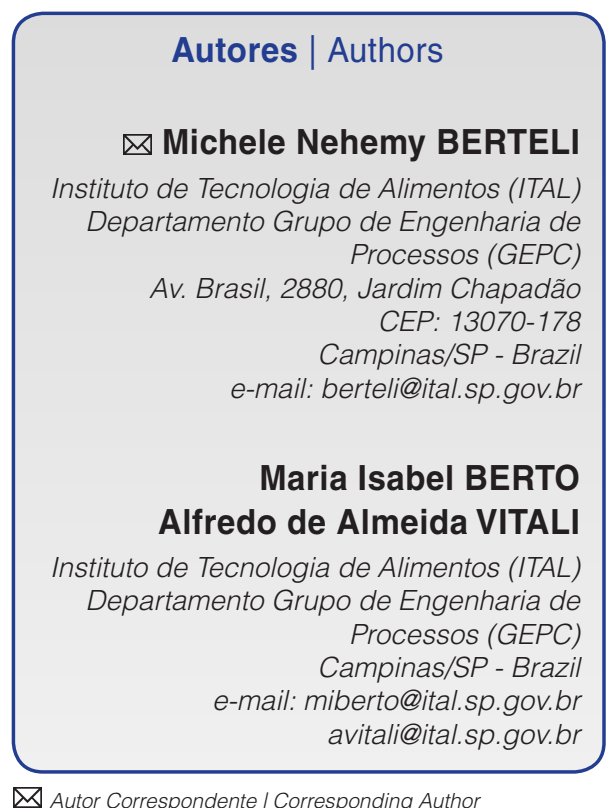

Recebido / Received: 15/05/2013 Aprovado / Approved: 15/08/2013

Publicado / Published: set./2013

\section{Resumo}

A letalidade, ou valor F, é uma grandeza utilizada para a representação do efeito do tratamento térmico nos alimentos e indica a intensidade desse tratamento em relação à morte de um micro-organismo alvo. O Método de Ball é muito útil e versátil, e a partir de parâmetros de penetração de calor, $j_{h}$ e $f_{h}$, específicos para cada produto, permite o cálculo da intensidade de um processo térmico de esterilização em autoclaves. O objetivo deste estudo foi verificar a aplicabilidade do Método de Ball para o cálculo do valor $F_{0}$ em produtos esterilizados em uma autoclave a vapor desaerada por água. Essa desaeração é um procedimento alternativo e baseia-se na expulsão do ar através do enchimento do equipamento com água. Os valores de $F_{0}$ obtidos pelo Método de Ball nos processos com desaeração por água e desaeração convencional foram comparados com os valores de $F_{0}$ calculados para os processos através do Método Genérico. Os resultados dos valores de $F_{0}$ calculados pelo método de Ball para os processos utilizando desaeração por água sempre foram inferiores aos obtidos pelo Método Genérico. As diferenças nos valores de $F_{0}$ entre as duas metodologias de cálculo foram semelhantes quando se compararam os processos de desaeração por água e convencional, confirmando a aplicabilidade do Modelo de Ball em processos de esterilização em autoclave a vapor desaerada por água.

Palavras-chave: Termoprocessamento; Método de Ball; Letalidade; Autoclave; Clostridium botulinum.

\section{Summary}

Lethality or the $\mathrm{F}$ value is a measure used to represent the effect of thermal treatment on foods, and denotes the intensity of such a treatment as concerns the death of a certain target microorganism. The Ball method is very useful and versatile in calculating the intensity of a thermal sterilization process carried out in retorts, based on the heat penetration parameters, jh and fh, specific for each product. The objective of this study was to verify the applicability of the Ball method in calculating the $F_{0}$ values for products sterilized in steam retorts, previously vented using water. Such venting is an alternative procedure based on expelling the air by filling the equipment with water. The $F_{0}$ values calculated for both venting processes (water and conventional) using the Ball method, were compared with the $F_{0}$ values calculated using the Generic Method. For the processes vented using water, the FO values calculated using the Ball method have always been lower than those obtained by Generic method. The differences in $F_{0}$ value obtained between the two venting processes (water and conventional) using the two calculation methods were similar, confirming the applicability of the Ball method in sterilization processes in water vented steam retorts.

Key words: Thermal processing; Ball method; Lethality; Retort; Clostridium botulinum. 
BERTELI, M. N. et al.

\section{Introdução}

Um processo térmico visa a garantir as qualidades sensoriais e, principalmente, a segurança microbiológica do alimento. Esta última pode ser avaliada através do cálculo da letalidade do processo (F), expressa em minutos e calculada baseando-se em uma temperatura de referência.

A partir dos históricos da temperatura do alimento no ponto de aquecimento mais lento, da temperatura da autoclave e do tempo de processo, o cálculo de um processo térmico pode ser efetuado através de alguns métodos, sendo os mais utilizados o Método Genérico e o Método Matemático de Ball. Os dois métodos são baseados em princípios idênticos, o da somatória das taxas letais, mas com procedimentos diferentes.

O Método Genérico foi o ponto de partida para uma abordagem científica de alimentos enlatados; é um método exato, baseado na somatória das taxas letais obtidas das curvas reais de penetração do produto. Entretanto, é limitado, no que diz respeito à simulação de processos térmicos. A partir do método desenvolvido por Ball (1923), não apenas a rotina de cálculos foi facilitada, como também passou a ser possível a simulação. O Método de Ball foi o precursor de uma série de metodologias mais recentes, mas, em razão da sua versatilidade, é ainda amplamente usado na indústria e considerado um exemplo de uso inteligente da matemática em processos de alimentos (STOFOROS, 2010).

Autoclaves que operam sob pressão de vapor são de concepção mais antiga e ainda as mais utilizadas nas indústrias que processam alimentos envasados em recipientes metálicos. Para o perfeito funcionamento dessa classe de autoclaves, é necessário o uso de vapor puro, ou seja, isento de ar, como meio de aquecimento (GMA, 2007).

As etapas do processo de esterilização em autoclave sob pressão de vapor são: i) tempo de subida de temperatura (CUT), que contempla a desaeração e a pressurização da autoclave até a temperatura de processo; ii) esterilização, e iii) resfriamento.

A operação denominada desaeração tem por finalidade a remoção total do ar do interior do equipamento através de injeção de vapor, garantindo a uniformidade e a eficiência da esterilização. A eliminação do ar é fundamental para manter o mesmo valor do coeficiente de transferência de calor por convecção (h) e, assim, garantir a reprodutibilidade do processo. $O$ valor de $h$ para vapor com $6 \%$ de ar chega a diminuir esse coeficiente em dez vezes, quando comparado ao vapor puro (FELLOWS, 2009).

Nas operações de esterilização de alimentos com autoclaves sob pressão de vapor, a demanda de vapor deve ser dimensionada para suprir a necessidade dos picos de vapor. Esses picos ocorrem exatamente na fase de desaeração, na qual a demanda de energia é máxima, sendo necessária, posteriormente, pouca energia para manter a temperatura do processo (LOPEZ, 1981; SIMPSON et al., 2005; BERTELI et al., 2013). Assim, ainda que a desaeração seja de relativa curta duração, grandes quantidades de vapor são gastas, o que torna essa etapa onerosa para as indústrias do setor.

Uma abordagem alternativa, descrita por Berteli et al. (2012), está baseada na utilização de água para deslocamento do ar em substituição ao processo convencional de desaeração em autoclaves operadas sob pressão de vapor. Esse processo alternativo consiste basicamente em expulsar o ar do equipamento através de seu arraste pelo enchimento da autoclave com água aquecida e posterior esvaziamento da mesma pela parte inferior, utilizando uma bomba, e com vapor entrando pela parte superior da autoclave. A metodologia proposta para a desaeração da autoclave por água mostrou-se eficiente. A partir da quantificação da vazão de vapor, verificou-se que a desaeração alternativa chegou a apresentar redução de 50 \% da necessidade de vapor em relação à desaeração convencional (BERTELI et al., 2013).

O presente estudo visou verificar a aplicabilidade do modelo de Ball para o cálculo da letalidade $\left(F_{0}\right)$ em produtos convectivos e condutivos, esterilizados em autoclaves desaeradas por água.

\section{Material e métodos}

\subsection{Sistema autoclave - bomba - tanque}

A Figura 1 apresenta a montagem e a instrumentação de um sistema autoclave - bomba - tanque usado para o estudo da desaeração por água em uma autoclave a vapor vertical. Os testes foram realizados na planta piloto de operações unitárias do Grupo de Engenharia de Processos - GEPC - ITAL. Uma breve descrição do sistema é apresentada a seguir e detalhes do equipamento e da metodologia estão apresentados em Berteli et al. (2012, 2013).

Um tanque cilíndrico (TC) foi conectado à linha de água da rede [1] e à linha de vapor [5]. O controle da temperatura da água do tanque foi feito com uma válvula controladora de temperatura de ação direta (Spirax Sarco, modelo SB, tipo 128I) [7] e por um sensor de temperatura [8]. Uma bomba centrífuga (BC) (KSB Hydrobloc model C700 (0,75 HP, 3450 rpm) foi instalada entre a autoclave e o tanque, e conectada a ambos. A função da bomba foi auxiliar a saída da água da autoclave e retorná-la ao tanque, permitindo sua reutilização em outros processos de desaeração.

As temperaturas da autoclave e do tanque foram medidas por meio de um aquisitor de dados de 16 canais 
BERTELI, M. N. et al.

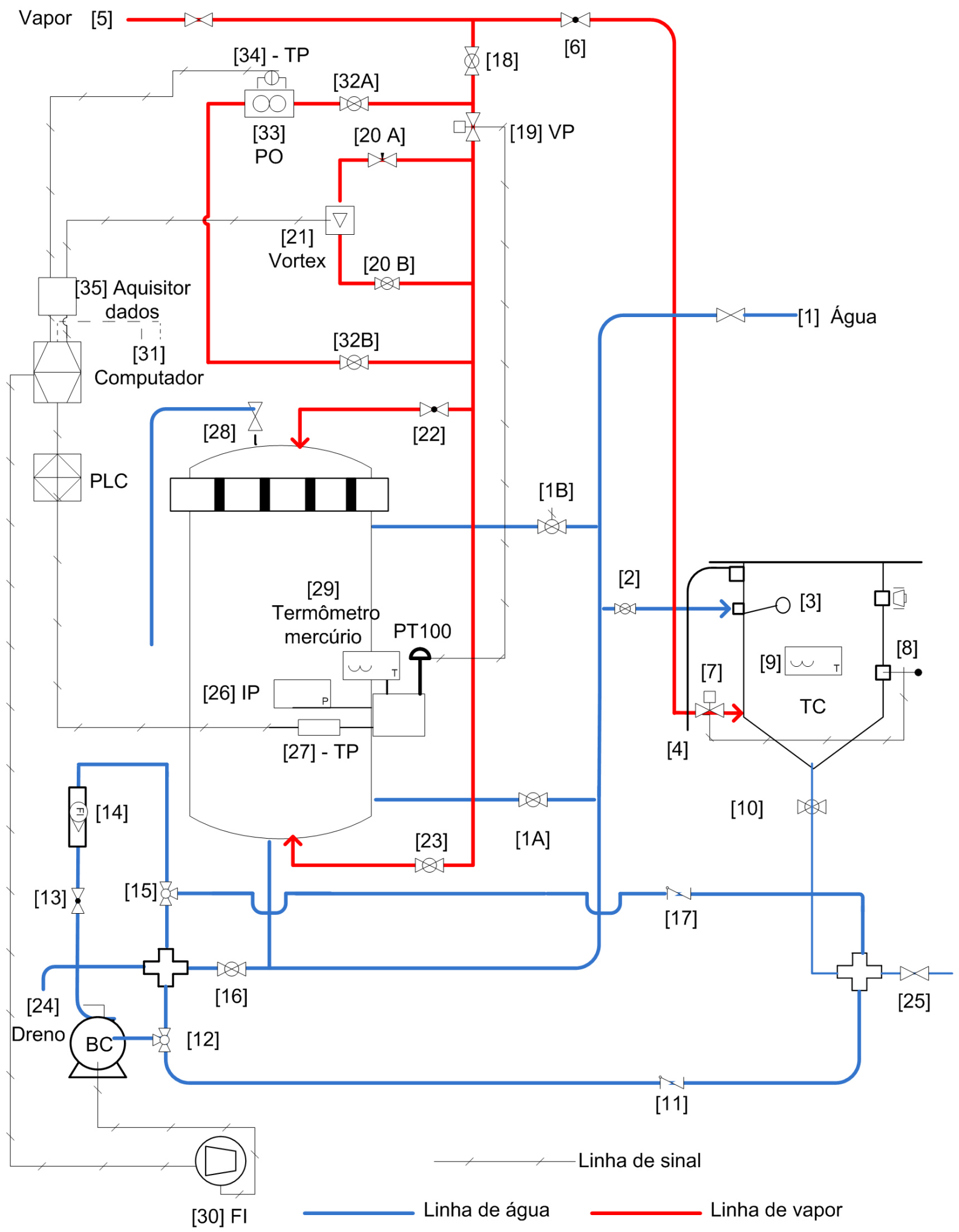

Figura 1. Esquema Sistema Autoclave - Bomba - Tanque: [1] linha de água (A) entrada pela parte superior da autoclave e (B) entrada pela parte inferior; [2] válvula esfera; [3] boia; [4] ladrão; [5] linha de vapor; [6] válvula esfera; [7] válvula controladora de temperatura por ação direta; [8] sensor de temperatura da válvula controladora; [9] termômetro; [10] válvula esfera; [11] válvula de retenção; [12] válvula de três vias; [13] válvula globo; [14] rotâmetro; [15] válvula de três vias; [16] válvula esfera; [17] válvula de retenção; [18] válvula esfera; [19] válvula posicionadora para controle da temperatura da autoclave (PV); [20] válvula agulha (A) e esfera (B); [21] medidor de vazão de vapor tipo Vortex; [22] válvula globo; [23] válvula esfera; [24] dreno da autoclave; [25] dreno do tanque; [26] manovacuômtero; [27] transmissor de pressão (PT); [28] válvula de desaeração e sangradores; [29] termômetro de mercúrio; [30] inversor de frequência (FI); [31] computador; [32A, 32B] válvulas esfera; [33] placa de orifício (OP); [34] transmissor de pressão (PT); [35] aquisitor de dados. 
BERTELI, M. N. et al.

e software E - Val TM Ver 2.00 ELLAB A/S, modelo TM 9616. A pressão dentro da autoclave foi monitorada por um manovacuômetro tipo Bourdon (IP), (Farmabrás) [26], posicionado no nicho da autoclave, e por um transmissor de pressão (PT) [27] (MBS 33, Danfoss), instalado no mesmo local.

Durante a etapa de desaeração, o fluxo de vapor passou por uma placa de orifício, calibrada para operar na faixa de 50 a $300 \mathrm{~kg} / \mathrm{h}$ (Valblock, NX0111) (PO) [33]. Após a desaeração, durante a etapa de esterilização, cuja vazão de vapor é bem menor, o fluxo de vapor passou por um medidor de vazão do tipo Vortex, calibrado para operar na faixa de 5 a $50 \mathrm{~kg} / \mathrm{h}$ (OVAL Smart EX DELTA, modelo VXW 1015 N526106A) [21].

Durante a esterilização, a temperatura da autoclave foi controlada por uma válvula posicionadora (VP) (Bürket, Positioner 1067) [19], conectada a um sensor de temperatura PT-100, posicionado no nicho da autoclave ao lado de um termômetro de mercúrio [29].

Os testes de esterilização na autoclave foram executados com procedimentos de desaeração por água e convencional, por arraste de vapor.

Para a desaeração por água, o seguinte procedimento foi adotado: no enchimento da autoclave, a água do tanque na temperatura estabelecida foi transferida pela bomba centrífuga (CP) para a autoclave, passando pela válvula [16], posicionada na parte inferior da autoclave. Para isto, a água passou pelas válvulas de três vias [12] e [15], respectivamente. Quando foi observado o transbordamento da água pelas válvulas de desaeração e sangradores [28], a válvula inferior da autoclave [16] e todas as válvulas de transbordamento foram fechadas e a bomba, desligada. Durante a etapa de desaeração, simultaneamente à saída da água da autoclave pela sua parte inferior, entrou-se com o vapor pela parte superior do equipamento. Para isto, a válvula de entrada de vapor pela parte superior da autoclave [22] e a válvula inferior da autoclave [16] foram abertas, e a bomba, religada. Assim, a água foi direcionada pela bomba para o tanque de estocagem pela válvula de três vias [12] posicionada no sentido autoclave-bomba e pela válvula de três vias [15] posicionada no sentido bombatanque de estocagem. Para evitar retornos na linha de água entre o tanque e a autoclave, duas válvulas de retenção [11 e 17] foram instaladas. Os ensaios foram realizados em duplicata com a água de desaeração nas temperaturas ambiente (cerca de $25^{\circ} \mathrm{C}$ ), $50{ }^{\circ} \mathrm{C}$ e $80^{\circ} \mathrm{C}$.

Já para a realização da desaeração convencional, o vapor entrou na autoclave pela sua parte inferior [válvula 23]. Todos os sangradores da autoclave foram mantidos abertos, assim como o desaerador [28], até que todos os termopares convergissem para a mesma temperatura. Os sangradores foram mantidos parcialmente abertos e o desaerador foi então fechado para a autoclave atingir a temperatura de set point e, assim, iniciar o processo de esterilização.

Os testes foram executados com a autoclave carregada com carga máxima. Para isto, foram fechadas, em uma recravadeira a vácuo (John Heine, modelo 71D, series 2), 150 latas do tipo para atum ( $83 \mathrm{~mm} \times 38 \mathrm{~mm}$ e espessura da folha $0,17 \mathrm{~mm}$ ) cheias de água (convectivo) e cheias de suspensão de Bentonita a 5 \% (condutivo) (BERTO et al., 2006), sem espaço livre. As latas foram distribuídas verticalmente em sete camadas, dentro de um cesto, em um arranjo vertical desalinhado, isto é, evitando-se uma perfeita sobreposição.

Para o acompanhamento das temperaturas da autoclave e do produto, sete termopares (TC) foram posicionados em diferentes pontos na autoclave e no interior de três latas, em diferentes alturas (Figura 2). Os TC 1, 2, 3 e 4 acompanharam as temperaturas da autoclave. Os TC 1, 2 e 3 foram fixados em latas dentro do cesto apenas com a estrutura lateral e com fundo e tampos removidos. O TC4 foi posicionado fora do cesto e abaixo do distribuidor de vapor da autoclave, representado pela linha tracejada da Figura 2. Os TC 5, 6 e 7 acompanharam a evolução das temperaturas dos produtos e, dessa forma, foram posicionados dentro das latas cheias de água (um terço da altura em relação ao fundo) ou de solução de bentonita (centro geométrico), e fixados com dispositivos do tipo poço.

Durante a execução dos experimentos, a autoclave foi alimentada por linha de vapor com 6 bar de pressão manométrica. A temperatura de processo programada durante a etapa de esterilização foi de $121^{\circ} \mathrm{C}$, com vapor saturado.

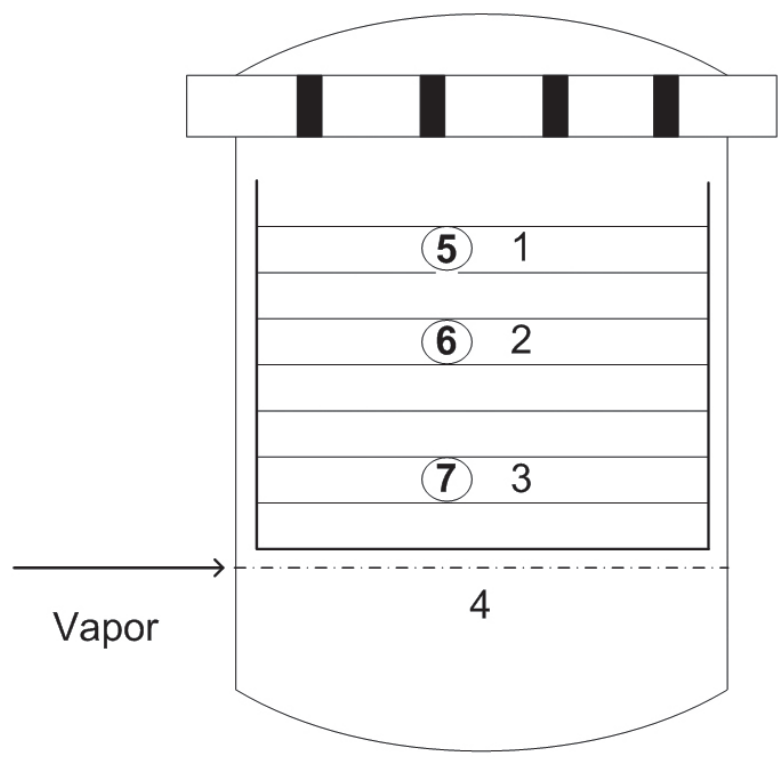

Figura 2. Distribuição dos termopares dentro da autoclave. Temperatura autoclave, TC 1, 2, 3 e 4; Temperatura produto, TC 5, 6 e 7 . 
BERTELI, M. N. et al.

\subsection{Cálculo do processo térmico}

O Método Genérico fornece os resultados mais reais nos cálculos dos processos térmicos e se aplica a qualquer tipo de processo, independentemente do mecanismo de aquecimento. A base experimental desse método é obter a curva de penetração de calor do produto no ponto mais lento de aquecimento em função do tempo e calcular a letalidade do processo a partir da integração da taxa letal (Equação 1), em função do tempo.

$$
\mathrm{TL}=10^{\left(\mathrm{T}-\mathrm{T}_{\mathrm{ref}}\right) / 2}
$$

Com a Equação 1, é possível determinar a taxa letal do processo a qualquer temperatura, desde que conhecido o valor $Z$ do micro-organismo em estudo. Sendo $\Delta$ t constante, o valor $F$ é a somatória das contribuições letais de cada estágio de temperatura em todo o processo (Equação 2).

$$
F=\sum T L \Delta t
$$

em que: $\Delta t=$ intervalo de tempo em cada temperatura (min.); $\mathrm{T}_{\text {ref }}=$ temperatura de referencia $\left({ }^{\circ} \mathrm{C}\right)$; Valor $\mathrm{Z}$ = parâmetro de letalidade do micro-organismo alvo a ser destruído (min.); $T$ = temperatura do ponto mais lento de aquecimento do produto $\left({ }^{\circ} \mathrm{C}\right)$.

Ball (1923) desenvolveu um modelo matemático muito útil e versátil para o cálculo da penetração de calor e da letalidade de um processo de esterilização, e definiu dois parâmetros para descrever a transferência de calor: fator de tempo, $f_{h}$, e fator de atraso de temperatura, $j_{h}$. A partir desses dois parâmetros de penetração de calor, $f_{h} e$ $\mathrm{j}_{\mathrm{h}}$, específicos para cada produto, processo e embalagem, o Método de Ball reproduz históricos de temperatura do produto em função do tempo (Equação 3).

$$
\log \left(T_{a}-T\right)=-t_{\text {BALL }} / f_{h}+\log \left[j_{h} \cdot\left(T_{a}-T_{0}\right)\right]
$$

em que: $\mathrm{T}_{\mathrm{a}}=$ temperatura do meio de aquecimento (autoclave) $\left({ }^{\circ} \mathrm{C}\right.$ ); $\mathrm{T}=$ temperatura do produto durante $\mathrm{o}$ aquecimento $\left({ }^{\circ} \mathrm{C}\right) ; \mathrm{T}_{0}=$ temperatura inicial do produto $\left({ }^{\circ} \mathrm{C}\right) ; \mathrm{t}_{\mathrm{BALL}}=$ tempo de Ball (min), que consiste no intervalo de tempo em que a autoclave trabalha à temperatura de processo, acrescido de $42 \%$ do tempo de subida de temperatura da autoclave (CUT); $f_{h}=$ fator de tempo (min), obtido da curva de aquecimento; $j_{h}=$ fator de atraso, adimensional, obtido da curva de aquecimento.

O parâmetro U é tempo de morte térmica do microorganismo à temperatura de processo, Ta (Equação 4). É uma função de $\mathrm{f}_{\mathrm{h}}$ e da diferença de temperatura entre aquela do meio de aquecimento $(\mathrm{Ta})$ e a do produto, no final do período de aquecimento $\left(\mathrm{T}_{\mathrm{g}}\right)$, definida como $\mathrm{g}$.

$U=10^{\left(T-T_{a}\right) / 2}$
$T_{g}=$ temperatura do produto no final do aquecimento $\left({ }^{\circ} \mathrm{C}\right) ; \mathrm{g}=$ diferença entre $\mathrm{T}_{\mathrm{a}}-\mathrm{T}_{\mathrm{g}}$.

Através de tabelas (STUMBO, 1973) ou equações que relacionam $U$ com f $_{h}$ e g (VINTERS et al.,1975), a letalidade de um processo térmico é calculada de acordo com a Equação 5.

$\frac{F_{0}}{U}=10^{\left(T_{a}-T_{\text {ref }}\right) / z}$

A avaliação da adequação do Modelo Matemático de Ball para o processo térmico na autoclave desaerada por água foi baseada na comparação da diferença dos valores de $\mathrm{F}_{0}$ obtidos pelo Método Genérico e Matemático, em ambos os processos de esterilização, ou seja, com a autoclave desaerada convencionalmente e por água, tanto para produto condutivo como convectivo.

\section{Resultados e discussão}

\subsection{Cálculos do processo térmico - testes condutivos e convectivos}

Na Tabela 1, estão descritos o tipo de desaeração, o produto, a temperatura da água de desaeração e os tempos obtidos durante os ensaios condutivos e convectivos realizados na autoclave a vapor vertical.

A partir dos históricos de temperatura da autoclave e do produto de cada processo, a letalidade foi calculada pelo Método Genérico e pelo Modelo Matemático de Ball. O micro-organismo de referência foi o $C$. botulinum $\left(\mathrm{T}_{\text {ref }}=121^{\circ} \mathrm{C}\right.$ e $\left.\mathrm{z}=10^{\circ} \mathrm{C}\right)$.

Os históricos de temperatura referentes aos processos com a autoclave desaerada por água a $80^{\circ} \mathrm{C}$ estão apresentados nas Figuras 3 e 5, e por vapor, nas Figuras 4 e 6, carregada tanto com produto condutivo como convectivo.

No processo a partir da desaeração convencional, o tempo de desaeração foi verificado quando os sensores de temperaturas TC1, TC2, TC3 e TC4 convergiram para a mesma temperatura $\left(\mathrm{T}_{\mathrm{a}}=121^{\circ} \mathrm{C}\right)$. No processo usando-se desaeração por água, foi considerado como desaeração o período entre o início de entrada de vapor na autoclave (início do esvaziamento) até o total preenchimento do equipamento com vapor (final do esvaziamento), quando também os sensores de temperaturas TC1, TC2, TC3 e TC4 convergiram para a mesma temperatura.

A discussão sobre os perfis e históricos de temperatura da autoclave e do produto durante a desaeração por água e por vapor foi apresentada por Berteli et al. (2013).

O $F_{0}$ obtido pelo método Genérico foi calculado pelo próprio aquisitor de temperatura Ellab, a partir das Equações 1 e 2. 
Aplicabilidade do método de Ball para o cálculo da letalidade de processos de esterilização em autoclaves a vapor desaeradas por água

BERTELI, M. N. et al.

Tabela 1. Nomenclatura, condições e tempos dos testes condutivos e convectivos.

\begin{tabular}{|c|c|c|c|c|c|c|}
\hline Teste & Produto & Desaeração & $\mathbf{T}_{\text {água }}\left({ }^{\circ} \mathbf{C}\right)$ & $t_{\text {des }}(m i n)$ & $t_{\text {CUT }}$ (min) & $t_{\text {processo }}(\mathrm{min})$ \\
\hline 1CCond & Condutivo & Vapor & -- & 3,7 & 5,3 & 32,4 \\
\hline 2CCond & Condutivo & Vapor & -- & 3,8 & 5,6 & 30,9 \\
\hline 1ACond & Condutivo & Água & 25 & 5,9 & 7,1 & 32,2 \\
\hline 2ACond & Condutivo & Água & 25 & 5,7 & 6,8 & 36,6 \\
\hline 3ACond & Condutivo & Água & 50 & 3,5 & 5,3 & 32,4 \\
\hline 4ACond & Condutivo & Água & 50 & 3,8 & 5,8 & 31,7 \\
\hline 5ACond & Condutivo & Água & 80 & 2,4 & 3,4 & 32,9 \\
\hline 6ACond & Condutivo & Água & 80 & 2,4 & 3,5 & 34,5 \\
\hline 1CConv & Convectivo & Vapor & -- & 4,6 & 6,3 & 7,9 \\
\hline 2CConv & Convectivo & Vapor & -- & 4,1 & 5,8 & 12,4 \\
\hline 1AConv & Convectivo & Água & 25 & 6,4 & 7,8 & 7,2 \\
\hline 2AConv & Convectivo & Água & 25 & 5,7 & 7,1 & 8,5 \\
\hline 3AConv & Convectivo & Água & 50 & 4,5 & 5,8 & 8,5 \\
\hline 4AConv & Convectivo & Água & 50 & 4,6 & 6,1 & 7,3 \\
\hline 5AConv & Convectivo & Água & 80 & 2,6 & 4,1 & 8,1 \\
\hline 6AConv & Convectivo & Água & 80 & 2,9 & 4,2 & 7,6 \\
\hline
\end{tabular}

$\mathrm{T}_{\text {água }}=$ temperatura da água de desaeração; $\mathrm{t}_{\text {des }}=$ tempo de desaeração; $\mathrm{t}_{\text {cut }}=$ tempo de CUT.

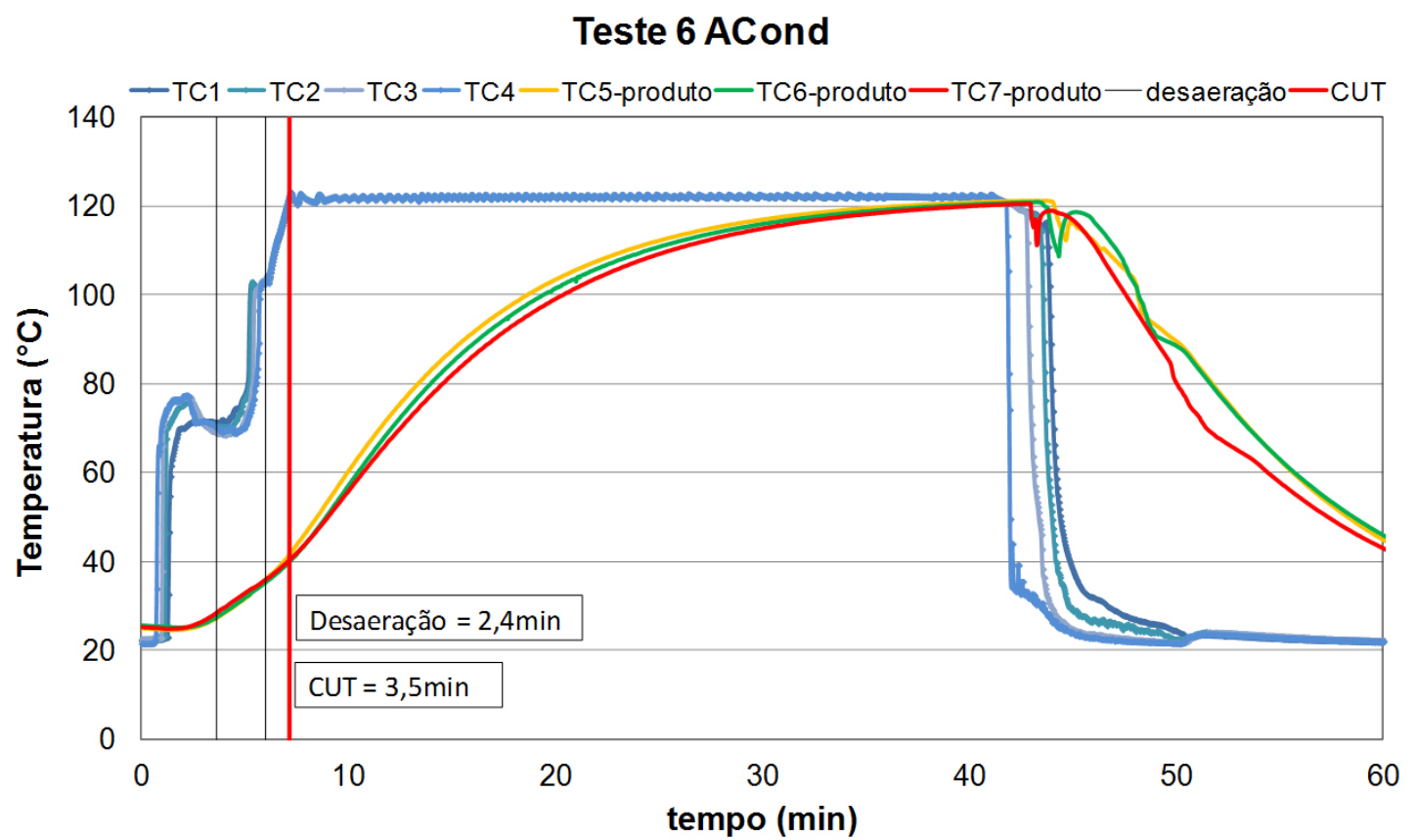

Figura 3. Históricos de temperatura: desaeração por água, $\mathrm{T}=80^{\circ} \mathrm{C}$, produto condutivo.

Para o método de Ball, foram levantados os parâmetros de letalidade $f_{h} e j_{h}$, a partir da curva de aquecimento do produto, e então aplicada uma rotina de cálculo, a partir das Equações 3 e 5 (VINTERS et al., 1975). Os parâmetros de letalidade sempre foram obtidos a partir da curva de temperatura referente ao TC posicionado na embalagem que resultou no menor valor de $\mathrm{F}_{\mathrm{o}}$, obtido pelo Método Genérico. O valor da temperatura da autoclave (Ta) usado foi a média dos valores registrados pelos 4 TCs (1, 2, 3 e 4) durante a etapa de esterilização, ou seja, após o CUT até o resfriamento.
Os resultados dos parâmetros $j_{h}$ e $f_{h}$, e dos valores dos coeficientes de correlação das curvas de aquecimento, além das diferenças entre o $F_{0}$ obtido pelo Método Genérico e aquele pelo Método de Ball, em minutos e em porcentagem referentes ao Fo Genérico, estão indicados na Tabela 2.

Verifica-se que os valores da letalidade obtidos pelo Método de Ball sempre foram inferiores aos obtidos pelo Método Genérico, confirmando a segurança deste método também em processos utilizando desaeração por água. 
Aplicabilidade do método de Ball para o cálculo da letalidade de processos de esterilização em autoclaves a vapor desaeradas por água

BERTELI, M. N. et al.

Teste 1 CCond

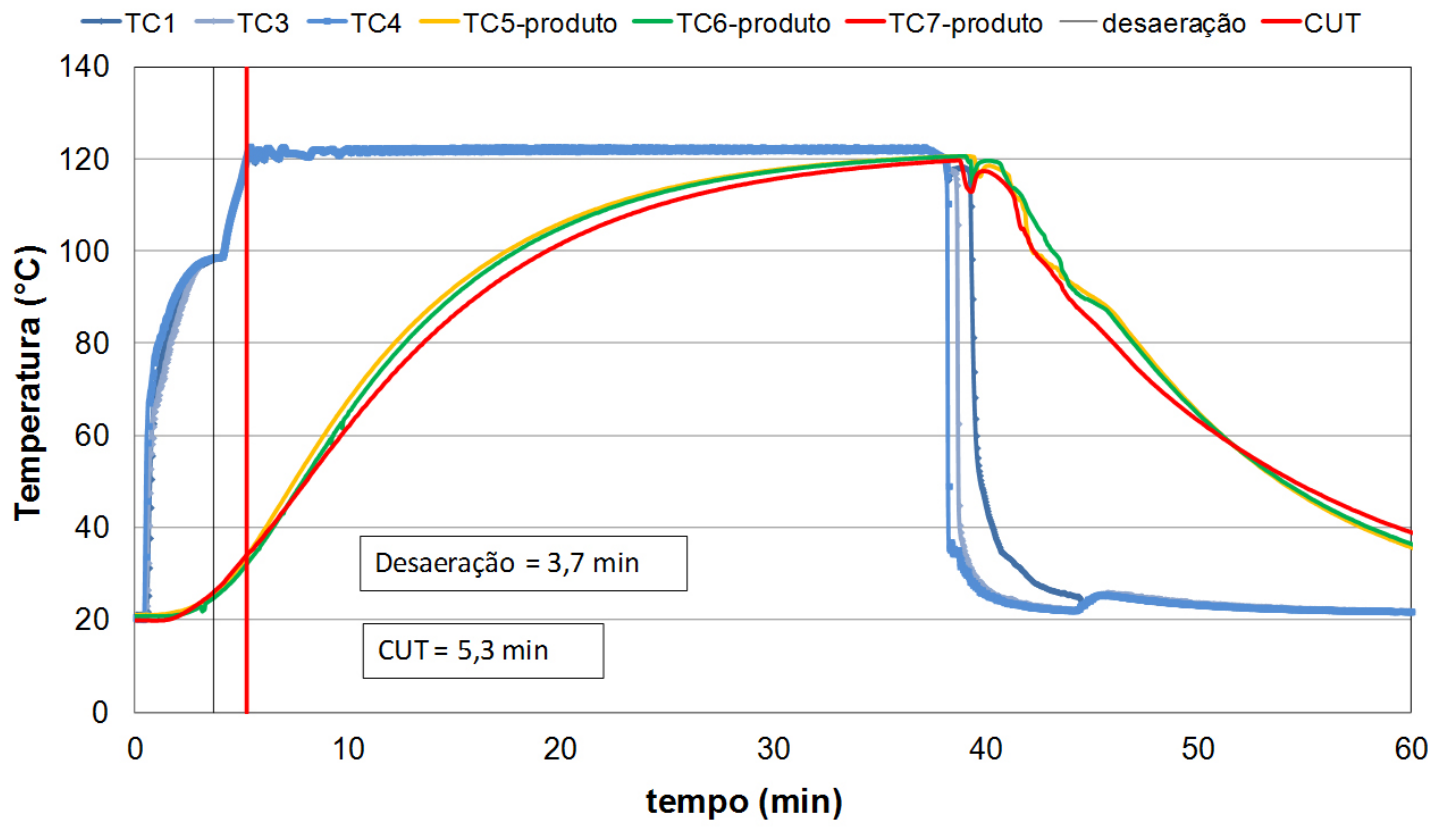

Figura 4. Históricos de temperatura: desaeração convencional, produto condutivo.

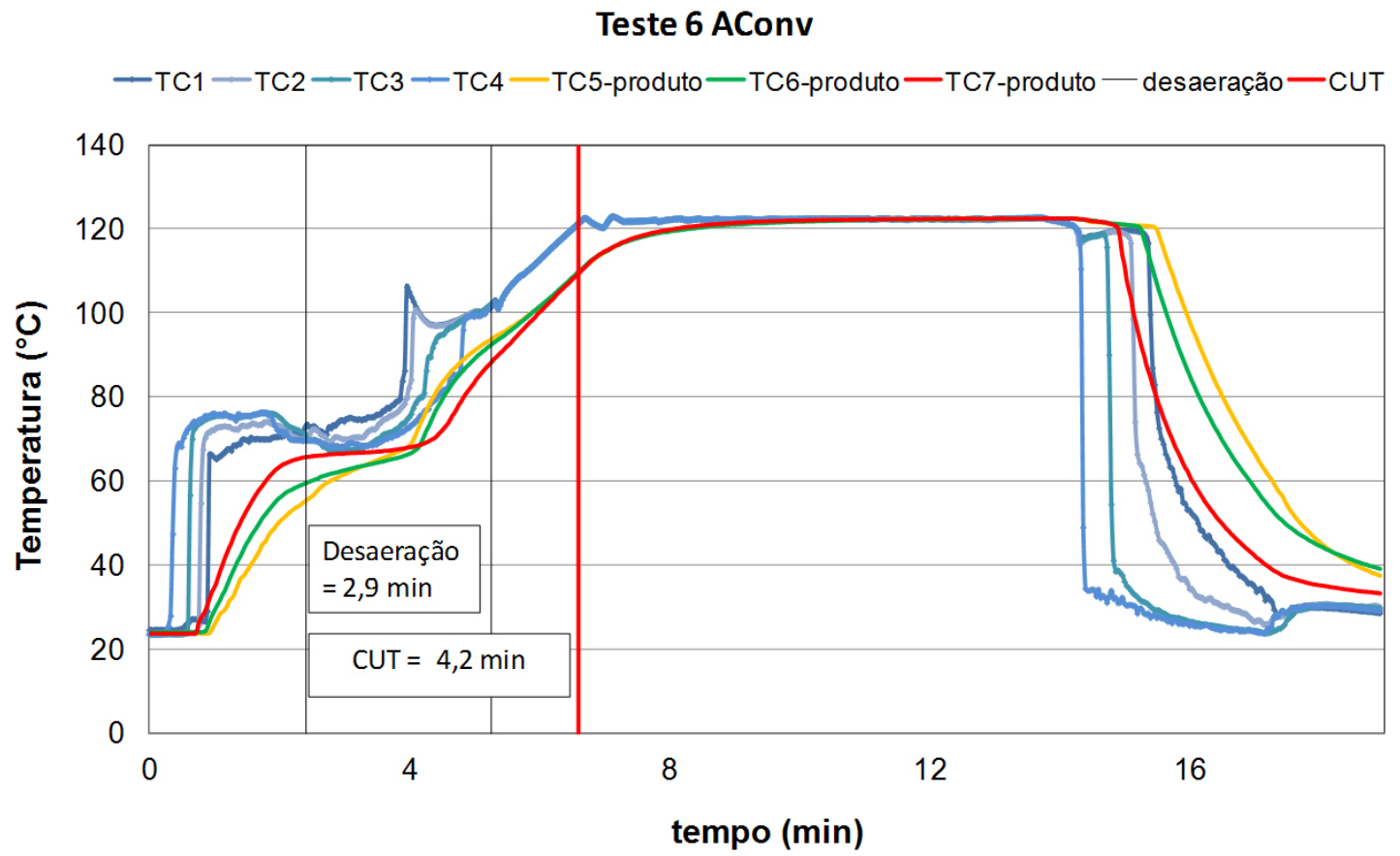

Figura 5. Históricos de temperatura: desaeração por água, $\mathrm{T}=80^{\circ} \mathrm{C}$, produto convectivo.

A diferença entre os resultados das duas metodologias de cálculo nos testes condutivos variaram de 4,2 a $12,6 \%$, nos teste 1 ACond e 6 ACond, respectivamente. Os valores de diferença percentual nos cálculos de $F_{0}$ mostraram-se semelhantes entre as duas metodologias de desaeração.
A diferença em valores reais foi maior nos ensaios

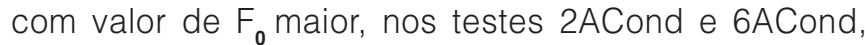
correspondendo às diferenças de 1,06 e 1,23 min, respectivamente. Para valores da mesma ordem de grandeza de $F_{0}$, nos testes 1CCond, 2CCond, 1ACond e 4ACond, as diferenças entre os resultados dos dois 
Aplicabilidade do método de Ball para o cálculo da letalidade de processos de esterilização em autoclaves a vapor desaeradas por água

BERTELI, M. N. et al.

Teste 1 CConv

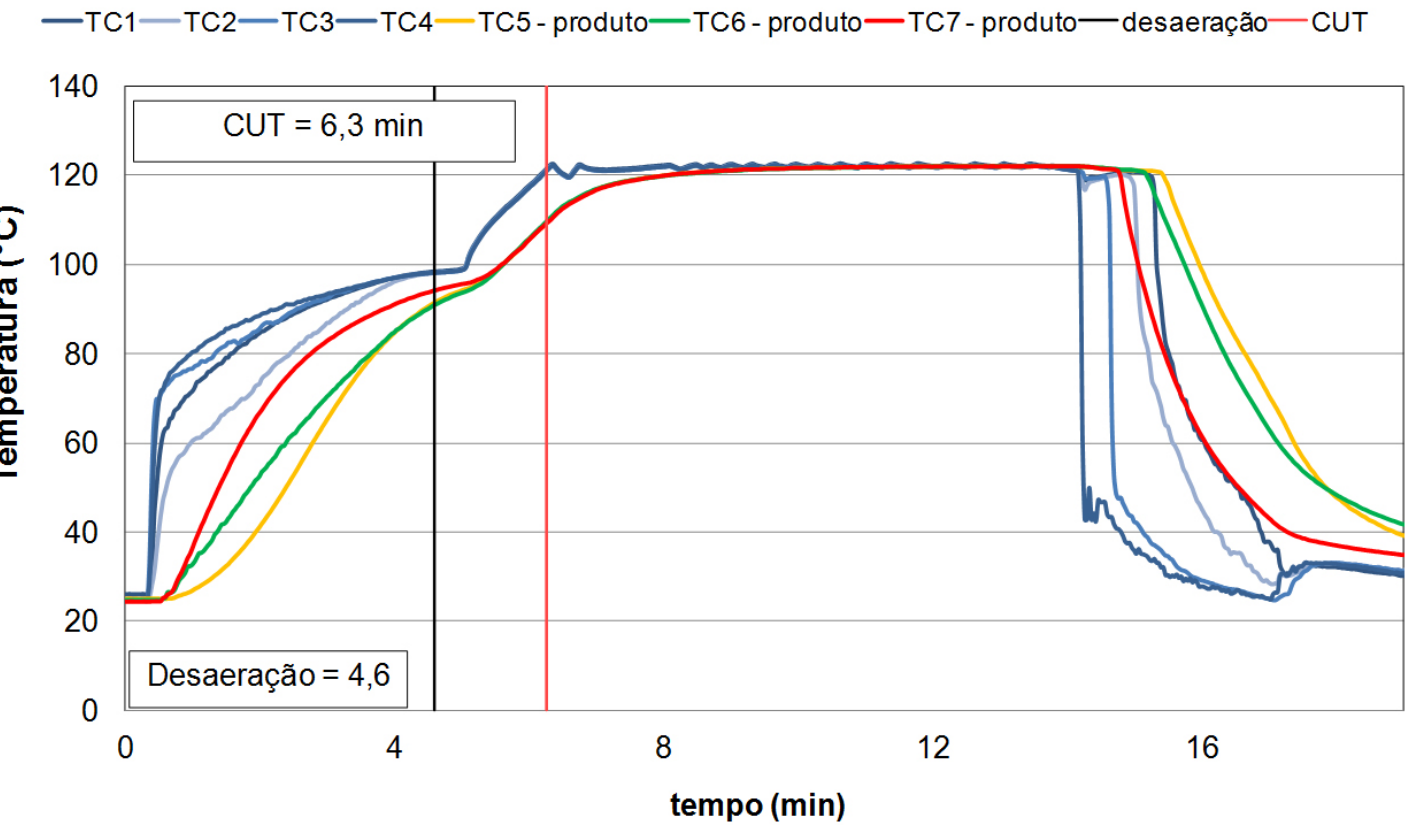

Figura 6. Históricos de temperatura: desaeração convencional, produto convectivo.

Tabela 2. Resultados dos cálculos de letalidade pelo Método Genérico e de Ball.

\begin{tabular}{|c|c|c|c|c|c|c|c|}
\hline \multicolumn{8}{|c|}{ Testes condutivos } \\
\hline Teste & $\mathrm{j}_{\mathrm{h}}$ & $f_{h}(\min )$ & $\mathbf{R}^{2}$ & $F_{0 \text { Ball }}(\min )$ & $F_{0 \text { Gen }}(\min )$ & $F_{0 \text { Gen }}-F_{0 \text { Ball }}(\mathrm{min})$ & $F_{0 \text { Gen }}-F_{0 \text { Ball }}(\%)$ \\
\hline 1CCond & 1,40 & 19,84 & 0,9995 & 5,77 & 6,41 & 0,64 & 10,0 \\
\hline 2CCond & 1,38 & 19,72 & 0,9996 & 5,77 & 6,20 & 0,43 & 6,9 \\
\hline 1ACond & 1,63 & 20,08 & 0,9994 & 5,72 & 5,97 & 0,25 & 4,2 \\
\hline 2ACond & 1,58 & 19,80 & 0,9993 & 8,94 & 10,00 & 1,06 & 10,6 \\
\hline 3ACond & 1,34 & 19,96 & 0,9996 & 6,31 & 7,13 & 0,82 & 11,5 \\
\hline 4ACond & 1,34 & 19,96 & 0,9996 & 6,15 & 6,67 & 0,52 & 7,8 \\
\hline 5ACond & 1,25 & 19,80 & 0,9991 & 7,66 & 8,56 & 0,90 & 10,5 \\
\hline 6ACond & 1,26 & 19,80 & 0,9991 & 8,50 & 9,73 & 1,23 & 12,6 \\
\hline \multicolumn{8}{|c|}{ Testes Convectivos } \\
\hline Teste & $\mathbf{j}_{\mathrm{h}}$ & $f_{h}(\min )$ & $\mathbf{R}^{2}$ & $F_{0 \text { Ball }}(\min )$ & $F_{0 G e n}(\min )$ & $F_{0 \text { Gen }}-F_{0 \text { Ball }}(\min )$ & $F_{0 \text { Gen }}-F_{0 \text { Ball }}(\%)$ \\
\hline 1CConv & 1,38 & 2,40 & 0,9982 & 7,75 & 8,46 & 0,71 & 8,4 \\
\hline 2CConv & 2,92 & 1,80 & 0,9955 & 14,91 & 15,54 & 0,63 & 4,0 \\
\hline 1AConv & 4,49 & 2,18 & 0,9972 & 7,34 & 8,74 & 1,40 & 16,0 \\
\hline 2AConv & 3,51 & 2,08 & 0,9964 & 8,56 & 9,84 & 1,28 & 13,0 \\
\hline 3AConv & 2,81 & 2,01 & 0,9987 & 9,83 & 10,86 & 1,03 & 9,5 \\
\hline 4AConv & 2,36 & 2,14 & 0,9969 & 8,06 & 9,06 & 1,00 & 11,0 \\
\hline 5AConv & 1,54 & 2,00 & 0,9955 & 8,35 & 9,11 & 0,76 & 8,3 \\
\hline 6AConv & 1,14 & 2,28 & 0,9983 & 8,27 & 8,99 & 0,72 & 8,0 \\
\hline
\end{tabular}

cálculos foram semelhantes entre si, independentemente do tipo de desaeração adotado, sendo 0,64; 0,43; 0,25; 0,52 min, respectivamente).

Já nos testes convectivos com desaeração por água, verificou-se que a diferença entre os valores reais e percentuais de $F_{0}$ obtidos pelas duas metodologias de cálculo decresceu com o aumento da temperatura da água de desaeração. Nos testes realizados com a autoclave desaerada por água à temperatura de $25^{\circ} \mathrm{C}$ (1AConv e 2AConv), as diferenças entre os valores de $\mathrm{F}_{0}$ obtidos pelo Método Genérico e o de Ball foram de 1,40 min (16\%) e 1,28 min (13\%); já nos teste realizados com a autoclave desaerada com água a $80^{\circ} \mathrm{C}$, a diferença dos valores obtidos foram de 0,76 min (8,3\%) e 0,72 min (8\%) (5AConv e 6AConv). Estes últimos ficaram próximos 
Aplicabilidade do método de Ball para o cálculo da letalidade de processos de esterilização em autoclaves a vapor desaeradas por água

BERTELI, M. N. et al.

Tabela 3. Resultados dos cálculos de letalidade no processo de desaeração por água considerando períodos distintos de desaeração: $\mathrm{t}_{\text {des }}[1]=$ tempo de esvaziamento da autoclave; $t_{\text {des }}$ [2] = tempo de enchimento e esvaziamento da autoclave.

\begin{tabular}{|c|c|c|c|c|c|}
\hline Testes & $F_{0}, t_{\text {des }}[1]$ & $F_{0}, t_{\text {des }}[2]$ & Testes & $F_{0}, t_{\text {des }}[1]$ & $\mathbf{F}_{0}, \mathbf{t}_{\mathrm{des}}[2]$ \\
\hline 1ACond & 5,72 & 5,71 & 1AConv & 7,34 & 7,33 \\
\hline 2ACond & 8,94 & 8,94 & 2AConv & 8,56 & 8,60 \\
\hline 3ACond & 6,31 & 6,31 & 3AConv & 9,83 & 9,81 \\
\hline 4ACond & 6,15 & 6,12 & 4AConv & 8,06 & 8,10 \\
\hline $5 A C$ ond & 7,66 & 7,64 & 5AConv & 8,35 & 8,25 \\
\hline 6ACond & 8,50 & 8,48 & 6AConv & 8,27 & 8,28 \\
\hline
\end{tabular}

aos valores de diferença de $F_{0}$ calculados nos testes de desaeração convencional.

A transferência de calor entre o meio e o produto é mais rápida, sob as mesmas condições, para produtos convectivos, em relação aos condutivos. Portanto, valores diferentes de CUT, intrínsecos às diferentes temperaturas da água de desaeração, podem ter influenciado nos resultados convectivos em função dos curtos tempos de processo (Tabela 1).

Os cálculos de todos os processos foram efetuados a partir dos históricos de temperatura do produto monitorado TC7, pois foi o que resultou no menor valor de $F_{0}$ obtido pelo Método Genérico, indiferentemente do tipo de desaeração adotado. O TC7 foi o sensor posicionado na embalagem mais próxima da entrada de vapor (Figura 2), mas também o mais próximo da entrada de água. Dessa forma, durante o resfriamento, o produto da lata em que estava posicionado o TC7 atingiu a temperatura limite de poder letal $\left(100^{\circ} \mathrm{C}\right)$ antes dos demais, resultando no menor valor de $\mathrm{F}_{0}$.

De acordo com Ball (1923), o tempo e o perfil do CUT contribuem para o efeito letal do tratamento térmico. Na medida em que o processo a partir de desaeração por água é inovador, durante os cálculos foram levados em consideração dois períodos distintos referentes ao da desaeração: [1] na primeira condição, o tempo de desaeração foi determinado entre o início de entrada de vapor na autoclave (início do esvaziamento) até o total preenchimento do equipamento com vapor (final do esvaziamento); [2] na segunda condição, o tempo de desaeração foi considerado desde o tempo zero de processo até o total preenchimento do equipamento com vapor, ou seja, desde o início do enchimento da autoclave com água até o final do esvaziamento da mesma. Os valores obtidos de $F_{0}$ para os dois períodos distintos de desaeração, praticamente não apresentaram diferenças (Tabela 3).

Portanto, de acordo com os dados apresentados na Tabela 3, verificou-se que o período de desaeração, considerando ou não o tempo de enchimento da autoclave piloto com água, não alterou os valores da letalidade do processo. Em processos industriais, em razão dos maiores volumes de água e, consequentemente, maiores tempos de desaeração, essa condição precisa ser estudada.

\section{Conclusões}

De acordo com estudos realizados, concluiu-se que a letalidade em processos de esterilização em autoclaves sob pressão de vapor desaerada por água pode ser calculada pelo Método de Ball. Pequenas diferenças foram observadas entre os valores de $F_{0}$ obtidos pelas duas metodologias de cálculo em relação aos valores de $F_{0}$ nos testes condutivos e à temperatura da água de desaeração nos testes convectivos. Em todos os cálculos, os valores de $F_{0}$ obtidos pelo Método de Ball foram inferiores aos calculados pelo Método Genérico.

\section{Agradecimentos}

Os autores agradecem à FAPESP - Fundação de Amparo à Pesquisa do Estado de São Paulo.

\section{Referências}

BALL, C. O. Determining by methods of calculation, the time necessary to process canned foods. Bulletin of the National Research Council, Washington, v. 37, p. 9-76, 1923

BERTELI, M. N.; VITALI, A. A.; MARSAIOLI JUNIOR, A.; BERTO, $M$. I. The analysis of an alternative approach to the venting process in retort operating under steam pressure. Journal of Food Engineering, Oxford, v. 109, n. 3, p. 388-398, 2012. http:// dx.doi.org/10.1016/j.jfoodeng.2011.11.014

BERTELI, M. N.; VITALI, A. A.; BERTO, M. I.; MARSAIOLI JUNIOR, A. Alternative venting in steam retorts - an approach of energy saving in thermal processing. Chemical Engineering and Processing: Process Intensification, Lausanne, v. 70, p. 204-210, 2013. http://dx.doi.org/10.1016/j.cep.2013.03.020

BERTO, M. I.; ATALA D. I. P.; VITALI, A. A. Simulação on line do perfil de temperatura de produtos durante o processo de esterilização. In: CONGRESSO BRASILEIRO DE ENGENHARIA QUÍMICA, 16., 2006, Santos. Anais... Santos: EEL-USP, 2006. CD-ROM

FELLOWS, P. J. Heat processing. In: FELLOWS, P. J. Food Processing Technology: Principles and Practice. 3rd ed. Boca Raton: CRC Press, 2009. cap. 12, p. 339-429.

GROCERY MANUFACTURERS ASSOCIATION - GMA. Canned Foods - Principles of Thermal Process Control, 
Aplicabilidade do método de Ball para o cálculo da letalidade de processos de esterilização em autoclaves a vapor desaeradas por água

BERTELI, M. N. et al.

Acidification and Container Closure Evaluation. 7th. ed. Washington, 2007. 216 p.

LOPEZ, A. Basic information on canning. In: LOPEZ, A. A Complete Course in Canning. Baltimore: The caning trade Inc., 1981. v. 1, cap. 2, p. 24-42.

SIMPSON, R.; COTÉS, C.; TEIXEIRA, A. Energy consumption in bath thermal processing: model development and validation. Journal of Food Engineering, Oxford, v. 73, n. 3, p. 217-224, 2005. http://dx.doi.org/10.1016/j.jfoodeng.2005.01.040
STOFOROS, N. G. Thermal process calculations through Ball's original formula method: A critical presentation of the method and simplification of its use through regression equations. Food Engineering Reviews, New York, v. 2, n. 1, p. 1-16, 2010. http:// dx.doi.org/10.1007/s12393-010-9014-4

STUMBO, C. R. Thermobacteriology in Food Processing. 2nd ed. New York; London: Academic Press, 1973. 328 p.

VINTERS, J. E.; PATEL, R. H.; HALABY, G. A. Thermal process evaluation by programmable computer calculator. Food Technology Journal, Chicago, v. 29, n. 3, p. 44-48, 1975. 